\title{
Automatic Tuberculosis Screening using Chest Radiographs
}

\author{
S. Leopauline, R. Kalpana, P. Sharmila
}

\begin{abstract}
Tuberculosis is considered to be dreadful disease also became greater peril in many regions of the world. Demonising tuberculosis still remains a challenging process where Opportunistic infections in immune compromised HIV/AIDS patients. If it is left untreated, rate of patients with tuberculosis are huge. We have standard diagnostics methods which are not considered to be accurate.They are sluggish and un faithful. An effort towards detection of tuberculosis is made in this paper by automated approach using chest radio graphs. In this method primary step is to segment an image suing texture method and lung region is extracted using graph cut extraction method. For the above said method a set of texture and shape features are formed on the lung region to enable the CXR,then binary classifier is used to detect normal or abnormal images. In proposed method we use Artificial Neural Networks (ANN) for screening and to identify the presence of tuberculosis
\end{abstract}

Keywords: CXR, Texture segmentation, Graph cut extraction, ANN Network, Tuberculosis.

\section{INTRODUCTION}

Tuberculosis (TB) is a polluting illness which chiefly brought about by bar formed microscopic organisms called Mycobacterium tuberculosis(MTB).Tuberculosis is the infectious can spread through little beads which turns out from air by means of hacks and wheezes, which fundamentally influences the lungs of human and gradually influences every other piece of the body.

Nearly the contaminations of tuberculosis don't demonstrate any manifestations ,on the off chance that they demonstrate any it is called as inert tuberculosis. The manifestations of TB are a ceaseless hack with blood, containing sputum, fever, night sweats and weight loss. If the dormant is left untreated it might transform into dynamic malady and murders a large portion of those affected. Infection of different organs can cause a wide scope of side effects.

Tuberculosis is spread through the air when people who have dynamic TB in their lungs hack, spit, talk, or wheeze. People with inactive TB don't spread the disorder. Pollution happens even more much of the time in people with HIV/AIDS and in the people who smoke. Assurance of TB relies upon the tuberculin skin test (TST) or blood tests.

Revised Manuscript Received on December 30, 2019.

* Correspondence Author

S.Leopauline, Department of Electronics and Communication Engineering, Vel Tech Multi Tech Dr.Rangarajan Dr.Sakunthala Engineering College, Chennai, India

R.Kalpana, Department of Electronics and Communication Engineering, Vel Tech Multi Tech Dr.Rangarajan Dr.Sakunthala Engineering College, Chennai, India

P.Sharmila, Department of Electronics and Communication Engineering, Vel Tech Multi Tech Dr.Rangarajan Dr.Sakunthala Engineering College, Chennai, India

(C) The Authors. Published by Blue Eyes Intelligence Engineering and Sciences Publication (BEIESP). This is an open access article under the CC BY-NC-ND license (http://creativecommons.org/licenses/by-nc-nd/4.0/)
Anticipation of TB includes screening those at high hazard, early location and treatment of cases, and immunization with the bacillus Calmette-Guérin (BCG) antibody. Heinrich Hermann Robert Koch was a German doctor and microbiologist, as the organizer of present day bacteriology, distinguished and portrayed the bacillus causing tuberculosis, M. tuberculosis, on 24 March 1882. He got the Nobel Prize in physiology and drug in 1905 for this revelation.The Support vector machine (SVM)method which was utilized before to identify Tuberculosis The fundamental hindrance of the SVM calculation is that it has a few key parameters that should be set effectively to accomplish the best grouping outcomes for some random issue.

The Parameters that may bring about astounding order exactness for issue A, may bring about a poor grouping precision for issue $B$. The client may, thusly, need to try different things with various diverse parameter settings so as to accomplish an agreeable outcome. Also, at long last we intended to break down the screening of tuberculosis dependent on exactness and speed.

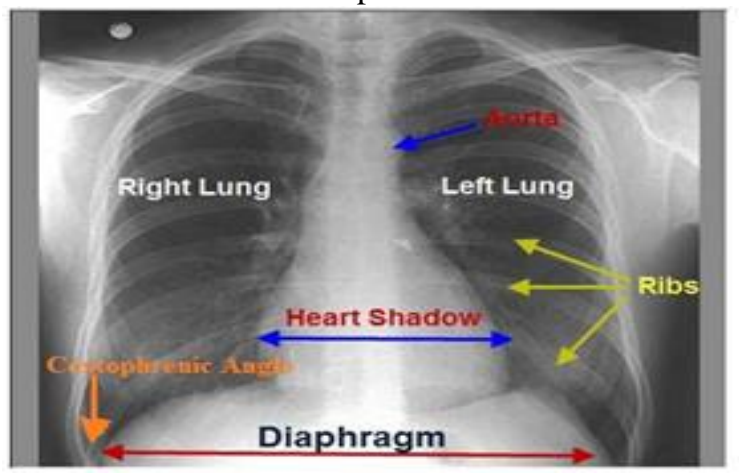

Fig.1: Lung Region

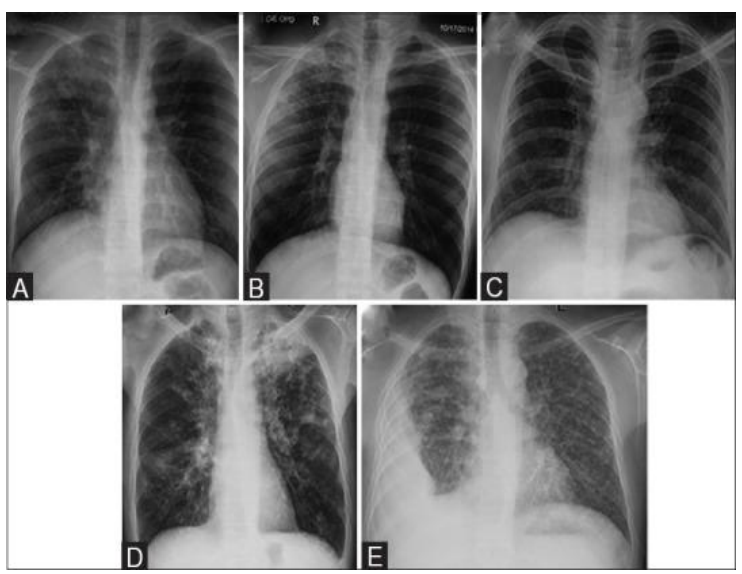

Fig. 2(A)CXR delineates RT upper zone union with noticeable Rt hilum.

Published By: 
(B)CXR in an different patient demonstrates multiple coalescent air-space knobs in RT upper zone. (C)CXR in an alternate patient ahows various not well characterized reticulo-nodular injuries in the two lungs with basa predominance,suggestive of military TB.

(D)CXR in an alternate patient shows dynamic postessential TB.

(E)There is RT-sided loculated pleural emanation with various air-space knobs dispersed in the two lungs.

\section{PROPOSED SYSTEM}

First, to segment the lung of the input CXR using Texture segmentation method. From the segmented lung field, a set of features like texture, shape, size is extracted using graph cut extraction. These extracted features are the inputs to binary classifier. Finally, using the extracted features outputs it confidence in classifying the input CXR as a TB positive case or not. For further classification we are using artificial neural network (ANN) to classify the stages.

\section{A. Texture Segmentation}

Surface division has for some time been a significant undertaking in picture handling. Essentially it targets sectioning a finished picture into a few districts having the comparable examples. A powerful and productive surface division technique will be helpful in applications like the examination of flying pictures, biomedical pictures and seismic pictures just as the mechanization of mechanical applications.

Surface element extraction strategies can be arranged into three significant classifications, in particular, measurable, auxiliary and ghastly. In measurable methodology, surface insights, for example, the snapshots of dim level histogram, or measurements dependent on dim level co-event lattice are figured to separate various surfaces.

\section{B. Graph Cut Extraction}

- Graph cut extraction-based methodologies are applied as worldwide streamlining strategies for PC vision issues, for example, picture extraction.

- The edges are cut and the careful arrangement can be found in polynomial time.

- It also minimizes the computation time and performance of the algorithm is satisfiable.

- The graph cut method is estimated to be high and used in multi-region image segmentation.

- Besides, clients can distinguish the item and foundation areas intelligently as topological requirements to improve the nature of extraction. Processing the images with ANN successfully resolves the problems of classification, identification, diagnostics, optimization and approximation

\section{Artificial Neural Networks (ANN)}

ANN computational model based on the structure and functions of biological neural networks.

The following diagram represents the general model of ANN followed by its processing:

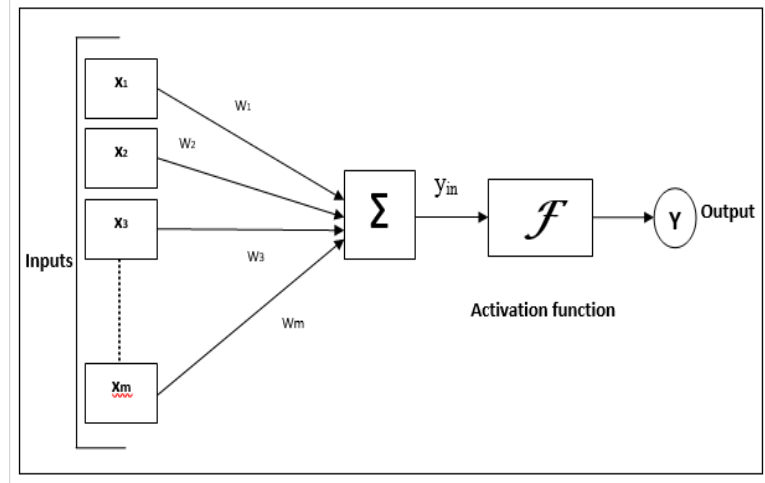

Fig.3: Model of ANN

For the above general model of counterfeit neural system, the net information can be determined as pursues

$$
\begin{gathered}
-\mathrm{Y} \text { in }=x 1 . w 1+x 2 . w 2+x 3 . w 3 \ldots x m . w m \\
\text { Yin }=x 1 . w 1+x 2 . w 2+x 3 . w 3 \ldots x m . w m
\end{gathered}
$$

i.e., Net input

$$
\text { yin }=\sum \text { mixi.wiyin }=\sum \text { imxi.wi }
$$

The output can be determined by applying the actuation work over the net information.

$$
\mathrm{Y}=\mathrm{F}(\mathrm{yin}) \mathrm{Y}=\mathrm{F} \text { (yin) }
$$

Yield $=$ work (net info determined)

ANNs have three layers that are interconnected. The primary layer contains information neurons. Those neurons send data on to the resulting layer, which in this manner sends the yield neurons to the third layer. Setting up a fake neural framework incorporates perusing allowed models for which there are a couple of related computations.

ANNs are seen as nonlinear truthful data showing instruments where the many-sided associations among information sources and yields are shown or models are found. ANN is generally called a neural framework. An ANN has a couple of focal points yet one of the most saw of these is the manner in which that it can truly pick up from viewing enlightening accumulations. Thusly, ANN is used as a discretionary limit gauge contraption.

\section{Feed Forward Network}

Feed forward systems are non-intermittent system having preparing units/hubs in layers and every one of the hubs in a layer are associated with the hubs of the past layers. The association has various loads upon them. There is no criticism circle implies the sign can just stream one way, from contribution to yield. It might be separated into the accompanying two sorts -

Single layer feed forward network - the idea is of feed forward ANN having just one weighted layer. As such, we can say the info layer is completely associated with the yield layer.

Multilayer feed forward network - the idea is of feed forward ANN having more than one weighted layer. As this system has at least one layers between the info and the yield layer, it is called concealed layers. 


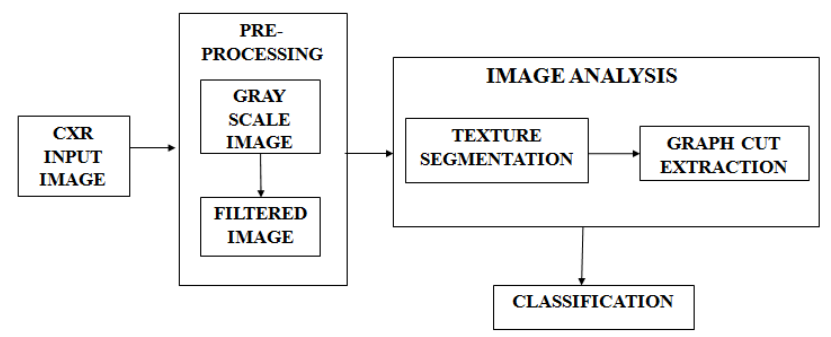

Fig.4:Work Flow

\section{RESULT AND DISCUSSION}

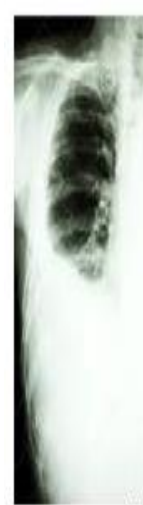

(a)
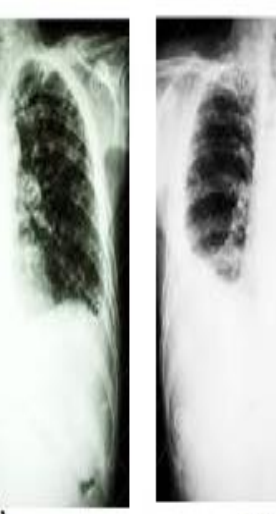

(b)

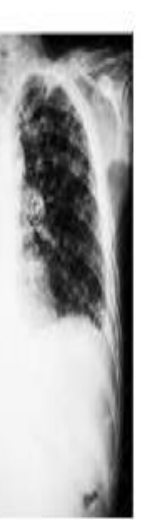

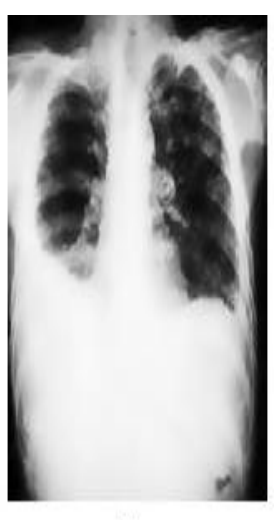

(c)
Fig 5(a) Input X-ray image (b).Gray scale image (c). Filtered image

In Fig 5a shows the chose $\mathrm{x}$-beam picture is given as the contribution to the MATLAB programming which plays out the activity of separating, fragmenting and recognizes the tuberculosis utilizing the chart cut extraction technique. In fig 5b shows info picture is first changed over into a dim scale picture, in which the estimation of every pixel is a solitary example speaking to just a measure of light, that is, it conveys just power data. This change dispenses with the shade and immersion data while holding the luminance. In fig 5c shows the sifting is a strategy for adjusting or improving a picture. The activity of sifting a picture handling incorporates smoothing, honing and edge improvement. The picture is sifted utilizing Gaussian channel which expels short commotion.
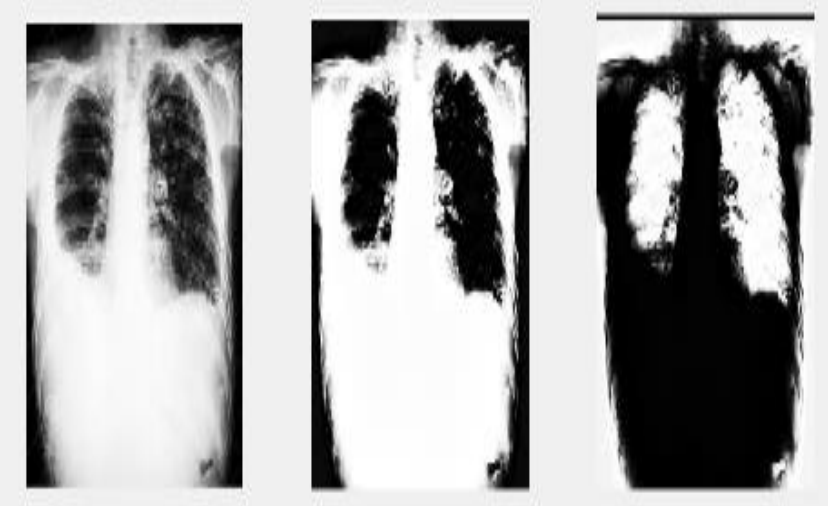

Fig.6:Lung Segmentation

Segmentation is an operation consisting in partitioning an image into multiple segments. Image segmentation is normally used to locate objects and boundaries that are lines, curves.

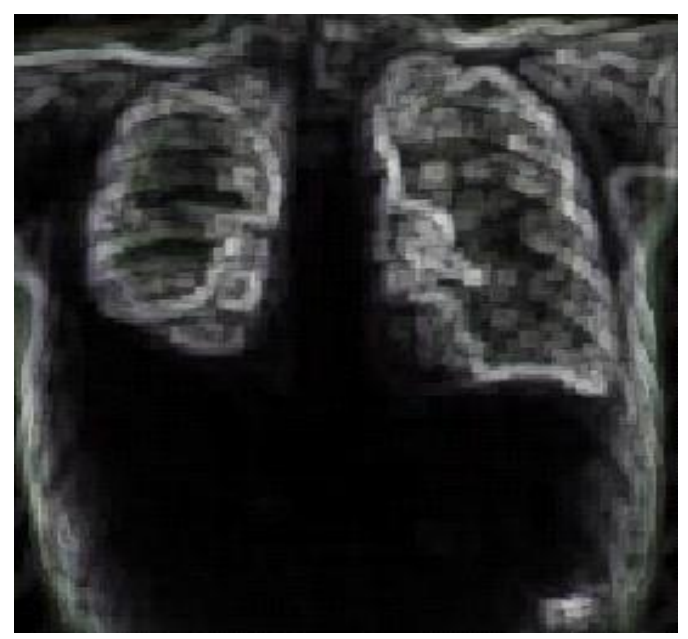

Fig.7: Texture Segmented Image
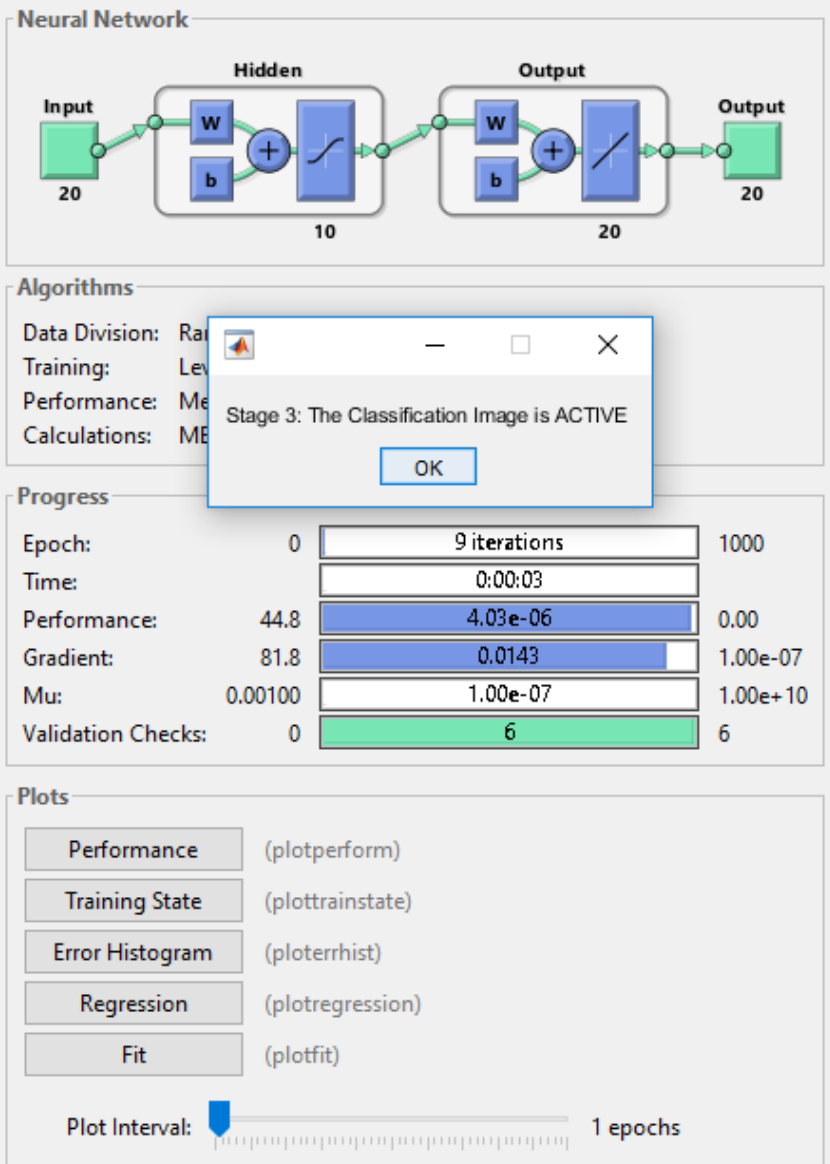

Fig.8: Stages Classification

MATLAB is a product bundle for superior numerical calculation and representation. It gives an intuitive condition with many implicit capacities for specialized calculation, designs and liveliness MATLABs worked in limits give phenomenal mechanical assemblies for direct polynomial math figuring's, data assessment, signal getting ready, progression, numerical courses of action of ODES, quadrature and various types of legitimate counts. By far most of these limits use the front line counts.

Published By:

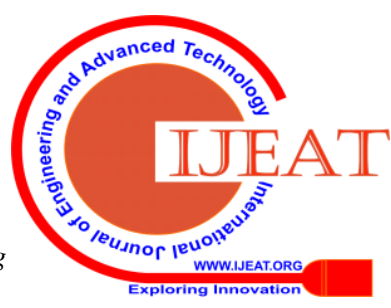


There are different capacities with regards to 2-D and 3-D course, MATLAB even gives an external interface to run those ventures from inside MATLAB.

\section{CONCLUSION}

State Vector Machine (SVM) is the technique is used in the detection of the Tuberculosis is less accurate and time consumption is also more as there is a need to combine the three masks. In order to overcome this drawbacks, we proposed the usage of graph-cut algorithm in the detection of tuberculosis and further classification is done by using ANN (Artificial Neural Network)

The proposed uses the graph cut extraction method on chest x-ray of the patient for the automatic detection of tuberculosis and further Artificial Neural Networks is applied to the image for the further clarification of tuberculosis based on its stages. The study shows, by the methods employed increases the accuracy of results obtained. This study also provides direction to further improve the detection techniques for automatic screening of tuberculosis using chest radiographs.

\section{FUTURE SCOPE}

Now a days, tuberculosis is increasing among the people due to the increase of HIV and bad habits in people. If not treated at the right time, tuberculosis will develop to next stage within the patient and it may also spread to person through air (by cough, sneezing..). Chest radiograph recognition systemshould be made progressively explicit so different highlights of lungs ought to be likewise be contemplated which can distinguish irregularities other than tuberculosis for example malignant growth, heart maladies and pneumonia. Also, the framework can be intended for different methods of tuberculosis screening too that incorporate smear microscopy and medication helplessness test.

\section{REFERENCES}

1. Ferani E. Zulvia, Kuo, R.J and Roflin, E (2017) "An Initial Screening Method for Tuberculosis Disease using a Multi-Objective Gradient Evolution -Based Support Vector Machine and C5.0 Decision Tree" IEEE DOI 10.1109/COMPSAC.2017.57

2. Muhammad Hammad Menon and Muhammad Hunain Menon (2017) "Chest Radiographs TB Detection using Gabor Filter and Svm Classifier" IEEE Conference ID: 40353

3. Santhosh, K.C. and Sameer Antani (2017) "Automated Chest X-ray Screening: Can Lung Region Symmetry help to Detect Pulmonary Abnormalities", IEEE Transactions on Image Processing , DOI 10.1109/TMI.2017.2775636.

4. Bram Van Ginneken, Klaus Reither and Pragnya Maghuskae "A Novel Multiple-Instance Learning Based Approach to Computer Aided Detection of Tuberculosis on Chest X-rays" (2015) IEEE Transactions on Medical Imaging, Vol. 34, No. 1, DOI: 10.1109/TMI.2014.2350539.

5. Bram Van Ginneken, Grant Theron (2015) "Automatic Detection of Tuberculosis in Chest Radiographs using a combination of Textural, Focal and shape Abnormality analysis" (2015) IEEE Tansactions on Medical Imaging,DOI 10.1109/TMI.2015.2405761.

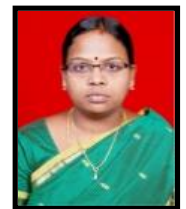

\section{AUTHORS PROFILE}

Ms S.Leopauline Assistant Professor,ECE Department,Vel Tech Multi Tech Engineering College, Chennai having teaching and research experience of more than 14 years.

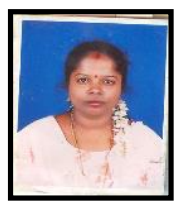

Ms. R.Kalpana Assistant Professor,ECE Department,Vel Tech Multi Tech Engineering College, Chennai having teaching and research experience of more than 16 years.

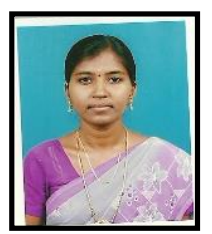

Ms. P.Sharmila Assistant Professor,ECE Department,Vel Tech Multi Tech Engineering College, Chennai having teaching and research experience of more than 10 years. 